\title{
Mappings preserving unit distance on Heisenberg group
}

\author{
J.M. Rassias ${ }^{*, 1}$, A. Charifi ${ }^{2}$, Ab. Chahbi ${ }^{3}$ and S. Kabbaj ${ }^{4}$ \\ 1 National and Copodistrian University of Athens, Pedagogical Department, Section of Mathematics and \\ Informatics, 4, Agamemnonos Str., Aghia Paraskevi, Athens 15342, Greece \\ 2,3,4 Department of Mathematics, Faculty of Sciences, University of Ibn Tofail, Kenitra, Morocco \\ * Corresponding author \\ E-mail: ${ }^{1}$ jrassias@primedu.uoa.gr, Ioannis.Rassias@primedu.uoa.gr, jrass@otenet.gr, \\ ${ }^{2}$ charifi2000@yahoo.fr, ${ }^{3}$ ab-1980@live.fr, ${ }^{4}$ samkabbaj@yahoo.fr
}

\begin{abstract}
Let $H^{m}$ be a Heisenberg group provided with a norm $\rho$. A mapping $f: H^{m} \rightarrow H^{m}$ is called preserving the distance $n$ if for all $x, y$ of $H^{m}$ with $\rho\left(x^{-1} y\right)=n$ then $\rho\left(f(x)^{-1} f(y)\right)=n$. We obtain some results for the Aleksandrov problem in the Heisenberg group.
\end{abstract}

2010 Mathematics Subject Classification. 39B82. 44B20, 46C05

Keywords. Isometry, Lipschitz mapping, conservative distance.

\section{Introduction}

Posed around 1970, the Aleksandrov problem investigates an isometry by the preservation of some properties of distance [1]. Several studies have been established to this subject on different normed spaces. I quote in this connection, the studies made by H. Y. Chu, C. G. Park, W. G. Park in 2004 on linear 2-normed spaces [8], J. M. Rassias, S. Xiang, M. J. Rassias in 2007 on the Aleksandrov and triangle isometry Ulam stability problem [16], X.Y. Chen, M.M. Song in 2010 on linear n-normed spaces [7] and D. Wang, Y. Liu, M. Song in 2012 on non-Archimedean normed spaces [23]. For more details the reader may also study [[2]-[6], [9]-[13], [15, 17],[18]-[22]].

The purpose of our contribution, is an idea introduced by J. M. Rassias that consists to apply it here to study the Aleksandrov problem in a Heinsenberg group.

Following this Introduction, some preliminary notations are set in the second Section, as well as our main new results are investigated in the third Section, respectively.

\section{Preliminary}

In this section we fix notations and special vocabulary that will be used later in the document.

Let $m$ be a fixed nonzero integer number. The $m^{\text {th }}$ Heisenberg group $H^{m}$ is of course a near isomorphism $\mathbb{C}^{m} \times \mathbb{R}$ endowed with the following group law

$$
(z, t)\left(z^{\prime}, t^{\prime}\right)=\left(z+z^{\prime}, t+t^{\prime}+\operatorname{Im}\left\langle z, z^{\prime}\right\rangle\right),(z, t),\left(z^{\prime}, t^{\prime}\right) \in \mathbb{C}^{m} \times \mathbb{R}
$$

where $z=\left(z_{i}\right)_{1 \leq i \leq m}, z^{\prime}=\left(z_{i}^{\prime}\right)_{1 \leq i \leq m}$ and $\left\langle z, z^{\prime}\right\rangle=\sum_{i=1}^{m} z_{i}{\overline{z^{\prime}}}_{i}$, with identity element $(0,0)$ and an inverse given by $(z, t)^{-1}=(-z,-t)$. The dilation $\delta_{s}$, for $s>0$, acts on the Heisenberg group as $\delta_{s}(z, t)=\left(s z, s^{2} t\right)$ and is its automorphism. The homogeneous norm

$$
\rho(z, t)=\left(|z|^{4}+t^{2}\right)^{\frac{1}{4}}
$$


defines the Heisenberg metric $d_{\rho}$ via the formula

$$
d_{\rho}(x, y)=\rho\left(x^{-1} y\right), x, y \in H^{m}
$$

Observe that the Heisenberg metric is really a metric and not just a quasi-metric since,

$$
\rho(x y) \leq \rho(x)+\rho(y)
$$

for all $x, y \in H^{m}$ (see $[7,8]$ for instance). It is also known that the Heisenberg metric $d_{\rho}$ and the Carnot Caratheodory metric $d$ are equivalent; that is, there exists a constant $c>1$ such that $c^{-1} d(x, y) \leq d_{\rho}(x, y) \leq c d(x, y)$ for all $x, y \in H^{m}$.

\section{Main result}

Let us etablish in this section the main results of this paper. We note that, throughout this section $H^{m}$ designates a Heisenberg group with its norm $\rho$.

Definition 3.1. A mapping $f$ of $H^{m}$ on itself is called an isometry if

$$
\rho\left(f(x)^{-1} f(y)\right)=\rho\left(x^{-1} y\right)
$$

for all $x, y \in H^{m}$.

If a mapping $f$ of $H^{m}$ on itself is an isometry then the inverse mapping is an isometry of $H^{m}$ onto $H^{m}$.

Definition 3.2. A mapping $\mathrm{f}$ of $H^{m}$ on itself, satisfies the strong distance one preserving property (SDOPP) if and only if for all $x, y \in H^{m}$ with $\rho\left(x^{-1} y\right)=1$ it follows that $\rho\left(f(x)^{-1} f(y)\right)=1$.

Definition 3.3. A mapping $f: H^{m} \rightarrow H^{m}$ satisfies the strong distance $n$ preserving property (SDnPP) if only if for all $x, y \in H^{m}$ with $\rho\left(x^{-1} y\right)=n$ it follows that $\rho\left(f(x)^{-1} f(y)\right)=n$.

Definition 3.4. Let $H^{m}$ be a Heisenberg group. We call a mapping $f: H^{m} \rightarrow H^{m}$ Lipschitz mapping if there is a $K>0$ such that

$$
\rho\left(f(x)^{-1} f(y)\right) \leq K \rho\left(x^{-1} y\right)
$$

for any $x, y \in H^{m}$.

Definition 3.5. We call a mapping $f: H^{m} \rightarrow H^{m}$ locally Lipschitz mapping if there is a $K>0$ such that

$$
\rho\left(f(x)^{-1} f(y)\right) \leq K \rho\left(x^{-1} y\right)
$$

whenever $\rho\left(x^{-1} y\right) \leq 1$.

We consider in this paper only the Lipschitz constant $K \leq 1$.

In this paper we shall study, mappings satisfying the weaker assumption that they preserve distance $n$ in both directions, instead of isometries. We shall see that such mappings are not far from being isometries. Let us prove the following Lemma.

Lemma 3.6. Let $H^{m}$ the Heisenberg group. Suppose that $f: H^{m} \rightarrow H^{m}$ is a surjective mapping satisfying (SDOPP). Then $f$ is bijective. 
Proof. We shall show that $f$ is injective. Suppose that there exist $x$ and $y$ in $H^{m}$ with $x \neq y$ such that $f(x)=f(y)$. Since $\rho\left(x^{-1} y\right) \neq 0$, we can set

$$
z=x \delta \frac{1}{\rho\left(y^{-1} x\right)}\left(y^{-1} x\right)
$$

thus,

$$
\rho\left(x^{-1} z\right)=\rho\left(\delta \frac{1}{\rho\left(y^{-1} x\right)}\left(y^{-1} x\right)\right)=1 .
$$

Since $f$ verify $f$ preserves the unit distance, that $\rho\left(f(x)^{-1} f(z)\right)=1$. Now we will prove that $\rho\left(y^{-1} z\right) \neq 1$. Suppose on the contrary, that $\rho\left(y^{-1} z\right)=1$. We have $y^{-1} z=y^{-1} x \delta \frac{1}{\rho\left(y^{-1} x\right)}\left(y^{-1} x\right)$, by identification we can put $y^{-1} x=\left(x_{1}, t_{1}\right)$ and denote $\alpha=\frac{1}{\rho\left(y^{-1} x\right)}$. This implies that

$$
\rho\left(y^{-1} z\right)=\rho\left((1+\alpha) x_{1},\left(1+\alpha^{2}\right) t_{1}\right)=\left((1+\alpha)^{4}\left|x_{1}\right|^{4}+\left(1+\alpha^{2}\right)^{2} t_{1}^{2}\right)^{\frac{1}{4}} .
$$

Since $\rho\left(y^{-1} z\right)=1$ and $\alpha^{4}\left|x_{1}\right|^{4}+\alpha^{4} t_{1}^{2}=1$, so

$$
\left(1+4 \alpha^{3}+4 \alpha^{3}+2 \alpha^{2}\right)\left|x_{1}\right|^{4}+\left(1+2 \alpha^{2}\right) t_{1}^{2}=0
$$

and so $x_{1}=0$ and $t_{1}=0$, then $x=y$, which is a contradiction. Therefore $\rho\left(y^{-1} z\right) \neq 1$. Now, we get $1=\rho\left(f(x)^{-1} f(z)\right)=\rho\left(f(y)^{-1} f(z)\right)$. Since $f$ preserves the unit distance, that $\rho\left(y^{-1} z\right)=1$ which is a contradiction. Therefore $f$ is a injective and surjective mapping then $f$ is bijective.

Q.E.D.

The following theorem gives the $n$-distance preserving mapping in both directions

Theorem 3.7. Let $H^{m}$ the Heisenberg group. Suppose that $f: H^{m} \rightarrow H^{m}$ is a surjective mapping satisfying (SDOPP) such that

$$
\rho\left(x^{-1} y\right)<1 \text { if an only if } \rho\left(f(x)^{-1} f(y)\right)<1 .
$$

Then $f$ preserves the area $n$ for each $n \in \mathbb{N}$.

Proof. By Lemma $3.6 f$ is a injective and since $f$ is surjective mapping then $f$ is bijective. Both $f$ and $f^{-1}$ preserves the unit distance and verify 3.1. Now we will prove that $f$ preserves distance $n$ in both directions for any positive integer $n$. In the sequel we shall need the following notations:

$$
\begin{aligned}
B(x ; r) & =\left\{z: \rho\left(x^{-1} z\right) \leq r\right\} \\
B_{0}(x ; r) & =\left\{z: \rho\left(x^{-1} z\right)<r\right\}
\end{aligned}
$$

Let $x$ be an arbitrary vector in $H^{m}$ and $n$ any positive integer, $n>1$. Assume that $z \in B(x, n)$. We can find a sequence $x=x_{0}, \ldots, z=x_{n}$, such that

$$
\left.x_{i}=x_{i-1} \delta_{\frac{1}{\rho\left(x^{-1} z\right)}}\left(z^{-1} x\right)\right), i=1, \ldots, n .
$$

Then,

$$
\rho\left(x_{i-1}^{-1} x_{i}\right)=\rho\left(\delta_{\frac{1}{\rho\left(x^{-1} z\right)}}\left(z^{-1} x\right)\right)=1, i=1, \ldots, n .
$$


Since $f$ preserves the unit distance, that

$$
\rho\left(f(x)^{-1} f(z)\right) \leq n
$$

Consequently, we have

$$
f(B(x, n)) \subset B(f(x), n), n>1 .
$$

The same result can be obtained for $f^{-1}$. Hence,

$$
f(B(x, n))=B(f(x), n), n>1 .
$$

Since $f$ and $f^{-1}$ verify 3.1 then

$$
f\left(B_{0}(x, 1)\right)=B_{0}(f(x), 1) .
$$

hold for all $x \in H^{m}$. Now we will prove that

$$
f\left(B_{0}(x, n)\right)=B_{0}(f(x), n) .
$$

for all $x \in X$, and $n \in \mathbb{N}$. Let $z \in B_{0}(x, n)$ and consider a sequence $x=x_{0}, x_{1}, \ldots, x_{n}=z$ such that

$$
x_{i}=x_{i-1} \delta_{\frac{1}{n}}\left(z^{-1} x\right), i=1, \ldots, n .
$$

Then

$$
\rho\left(x_{i-1}^{-1} x_{i}\right)=\rho\left(\delta_{\frac{1}{n}}\left(z^{-1} x\right)\right)=1, i=1, \ldots, n .
$$

Since $f\left(B_{0}(x, 1)\right)=B_{0}(f(x), 1)$, that

$$
\rho\left(f(x)^{-1} f(z)\right)<n
$$

Consequently, we have

$$
f\left(B_{0}(x, n)\right) \subset B_{0}(f(x), n), n \in \mathbb{N} .
$$

The same result can be obtained for $f^{-1}$. Hence,

$$
f\left(B_{0}(x, n)\right)=B_{0}(f(x), n), n \in \mathbb{N} .
$$

Consequently

$$
f(B(x, n)) \backslash f\left(B_{0}(x, n)\right)=B(f(x), n) \backslash B_{0}(f(x), n), n \in \mathbb{N} .
$$

So $f$ preserve the area $n$ for each $n \in \mathbb{N}$.

Q.E.D.

We will study the problem of mappings which preserve unit distance is an isometry.

Lemma 3.8. If a mapping $f$ is locally Lipschitz, then $f$ is a Lipschitz mapping.

Proof. We may assume that $\rho\left(x^{-1} y\right) \geq 1$, then there is $n_{0} \in \mathbb{N}$ such that $\rho\left(x^{-1} y\right) \leq n_{0}$. Let $x=x_{0}=\left(y_{0}, t_{0}\right), x_{1}, \ldots, x_{n_{0}}=y$, such that

$$
x_{i}=x_{i-1} \delta_{\frac{1}{n_{0}}}\left(y^{-1} x\right), i=1, \ldots, n_{0} .
$$


Then

$$
\rho\left(x_{i-1}^{-1} x_{i}\right)=\rho\left(\delta_{\frac{1}{n_{0}}}\left(y^{-1} x\right)\right) \leq 1, i=1, \ldots, n .
$$

Since $f$ is locally Lipschitz, so

$$
\rho\left(f\left(x_{i-1}\right)^{-1} f\left(x_{i}\right)\right) \leq K \rho\left(x_{i-1}^{-1} x_{i}\right)
$$

consequently

$$
\rho\left(f(x)^{-1} f(y)\right) \leq K \rho\left(x^{-1} y\right)
$$

Q.E.D.

Theorem 3.9. Let $f: H^{m} \rightarrow H^{m}$ be a locally Lipschitz mapping with the Lipschitz constant $K \leq 1$. Assume that $f$ is a surjective mapping satisfying (SDOPP). Then $f$ is a isometry.

Proof. By Lemma 3.6 and 3.8, $f$ Lipschitz mapping with the Lipschitz constant $K \leq 1$ and $f$ is bejective.For $x, y \in X$, there are two cases depending upon whether $\rho\left(f(x)^{-1} f(y)\right)=0$ or not.

In the first case $\rho\left(f(x)^{-1} f(y)\right)=0$ equivalent to $f(x)=f(y)$. Since $f$ is injective so $x=y$ and so $\rho\left(x^{-1} y\right)=0$.

In the remaining case $\rho\left(f(x)^{-1} f(y)\right)>0$, there is an $n_{0} \in \mathbb{N}$ such that $\rho\left(f(x)^{-1} f(y)\right)<n_{0}$. Assume that $\rho\left(f(x)^{-1} f(y)\right)<\rho\left(x^{-1} y\right)$. Set $x=x_{0}, x_{1}, \ldots, x_{n_{0}}=y$, such that

$$
x_{i}=x_{i-1} \delta_{\frac{1}{n_{0}}}\left(f(y)^{-1} f(x)\right), i=1, \ldots, n_{0} .
$$

Then we obtain that

$$
\rho\left(x_{i-1}^{-1} x_{i}\right)=\frac{\rho\left(f(y)^{-1} f(x)\right)}{n_{0}}, i=1, \ldots, n_{0}
$$

Thus

$$
\begin{aligned}
\rho\left(x^{-1} y\right) & =\rho\left(\prod_{i=1}^{n_{0}} x_{i-1}^{-1} x_{i}\right) \\
& \leq \sum_{i=1}^{n_{0}} \rho\left(x_{i-1}^{-1} x_{i}\right) \\
& =\sum_{i=1}^{n_{0}} \frac{\rho\left(f(y)^{-1} f(x)\right)}{n_{0}} \\
& =\rho\left(f(x)^{-1} f(y)\right) .
\end{aligned}
$$

Which is a contradiction, hence $f$ is a isometry.

Q.E.D.

\section{References}

[1] A.D. Aleksandrov, Mappings of families of sets, Soviet Math. 11 (1970), 116-120. 
[2] M. Bavand Savadkouhi, M. Eshaghi Gordji, J. M. Rassias, and N. Ghobadipour, Approximate ternary Jordan derivations on Banach ternary algebras, Journal of Mathematical Physics 50, 042303, (2009).

[3] R.L. Bishop, Characterizing motions by unit distance invariance, Math. Magazine 46 (1973), 148-151.

[4] A. Bodaghi, Cubic derivations on Banach algebras, Acta Math. Vietnam., No 4, Vol.38 (2013), $517-528$.

[5] A. Charifi, Iz. El-Fassi, B. Bouikhalene, S. Kabbaj, On the approximate solutions of the Pexiderized Golab-Schinzel functional equation, Acta Universitatis Apulensis, No. 38/2014, pp. $55-66$.

[6] Ab. Chahbi, A. Charifi, B. Bouikhalene, S. Kabbaj, Operatorial approach to the nonArchimedean stability of a Pexider K-quadratic functional equation, Arab Journal of Mathematical Sciences, Available online 17 January 2014, doi:10.1016/j.ajmsc.2014.01.001.

[7] X.Y. Chen, M.M. Song, Characterizations on isometries in linear n-normed spaces, Nonlinear Anal 2010;72:1895-901.

[8] H.Y. Chu, C.G. Park, W.G. Park, The Aleksandrov problem in linear 2-normed spaces, J. Math. Anal. Appl. 289 (2004) 666-672.

[9] G.G. Ding, On isometric extensions and distance one preserving mappings, Taiwanese J. Math. 10 (1) (2006), 243-249.

[10] D. V. Isangulova, The class of mappings with bounded specific oscillation, and integrability of mappings with bounded distortion on Carnot groups, Sibirsk. Mat. Zh. 48 (2007), no. 2, 313-334; English translation in: Siberian Math. J. 48 (2007), no. 2, 249-267.

[11] D. V. Isangulova and S. K. Vodopyanov, Sharp geometric rigidity of isometries on Heisenberg groups, Mathematische Annalen (2013), 1-29.

[12] A.V. Kuzminykh, Mappings preserving the distance 1, Siberian Math. J. 20 (1979), 417421.

[13] Y.M. Ma, The Aleksandrov problem for unit distance preserving mappings, Acta Math. Sci. 20 (3) (2000), 359-364.

[14] Yumei Ma, Isometry on linear n-normed spaces, Annales Academi Scientiarum Fennic Mathematica, Vol. 39 (2014), 973-981.

[15] Y.M. Ma, J.Y. Wang, On the A.D. Aleksandrov problem of isometric mapping, J. Math. Res. Exposition 23 (4) (2003), 623-630.

[16] B. Mielnik, Th.M. Rassias, On the Aleksandrov problem of conservative distances, Proc. Amer. Math. Soc. 116 (1992), 1115-1118.

[17] J.M. Rassias, S. Xiang, M.J. Rassias, On the Aleksandrov and triangle isometry Ulam stability problem, Int. J. Appl. Math. Stat. 7 (2007), 133-142. 
[18] Th.M. Rassias, P. Semrl, On the Mazur-Ulam problem and the Aleksandrov problem for unit distance preserving mappings, Proc. Amer. Math. Soc. 118 (1993), 919-925.

[19] K. Ravi, E. Thandapani, B. V. Senthil Kumar, Solution and stability of a reciprocal type functional equation in several variables, J. Nonlinear Sc. Appl., 7 (2014), 18-27.

[20] K. Ravi, M. Arunkumar, P. Narasimman, Fuzzy stability of an additive functional equation, Int. Journ. Math. and Stat., Vol. 9, No A11 (2011), 88-105.

[21] G. Zamani Eskandani, Pasc Gavruta, John M. Rassias and Ramazan Zarghami, Generalized Hyers-Ulam stability for a general mixed functional equation in quasi--normed spaces, Mediterr. J. Math. 8 (2011), 331-348.

[22] Tian Zhou Xu, John Michael Rassias, and Wan Xin Xu, Intuitionistic fuzzy stability of a general mixed additive-cubic equation, Journal of Mathematical Physics 51, 063519, (2010).

[23] D. Wang, Y. Liu and M. Song, The Aleksandrov problem on non-Archimedean normed space, Arab Journal of Mathematical Sciences, 18(2) (2012), 135-140. 